Meta

Journal des traducteurs

Translators' Journal

\title{
Les locutions nominales dans les dictionnaires de spécialités
}

\section{Mariette Meunier-Crespo}

Volume 42, numéro 1, mars 1997

Lexicologie et terminologie

URI : https://id.erudit.org/iderudit/002436ar

DOI : https://doi.org/10.7202/002436ar

Aller au sommaire du numéro

Éditeur(s)

Les Presses de l'Université de Montréal

ISSN

0026-0452 (imprimé)

1492-1421 (numérique)

Découvrir la revue

Citer cet article

Meunier-Crespo, M. (1997). Les locutions nominales dans les dictionnaires de spécialités. Meta, 42(1), 68-71. https://doi.org/10.7202/002436ar

\section{Résumé de l'article}

L'auteur propose une approche micro-syntaxique des locutions nominales dans les dictionnaires de spécialité, qui doit comporter des procédures spécifiques pour l'identification de ces locutions. d'utilisation que vous pouvez consulter en ligne.

https://apropos.erudit.org/fr/usagers/politique-dutilisation/ 


\title{
LES LOCUTIONS NOMINALES DANS LES DICTIONNAIRES DE SPÉCIALITÉS*
}

\author{
MARIETTE MEUNIER-CRESPO \\ Université Jean Moulin Lyon III, Lyon, France
}

\begin{abstract}
Résumé
L'auteur propose une approche micro-syntaxique des locutions nominales dans les dictionnaires de spécialité, qui doit comporter des procédures spécifiques pour l'identification de ces locutions.
\end{abstract}

\begin{abstract}
For dealing with noun phrases in specialized dictionaries, the author proposes a microsyntactic approach comprising specific procedures for identiying these expressions.
\end{abstract}

Les dictionnaires de spécialités recensent majoritairement non pas des mots simples mais des locutions nominales, dont le statut théorique est toujours discuté, voire contesté. Nous proposons ici une approche «micro-syntaxique» qui nous semble propre à aider le travail du lexicographe.

\section{DESCRIPTION}

Une locution nominale de spécialité, c'est une création sémantique et formelle composée le plus souvent de deux éléments, un élément nominal principal et un élément nominal, adjectival ou prépositionnel satellisé :

(a) nom + nom : épargne logement, cité dortoir nom + adjectif : table ronde, axe piétonnier nom + syntagme prépositionnel : zone de chalandise, gel des salaires

Beaucoup plus rarement, elles apparaissent sous la forme:

(b) verbe + nom : lave-vaisselle, sèche-linge

Cependant, la nouveauté lexicale des langues de grande communication dans le domaine roman réside dans l'apparition massive des locutions nominales des types décrits en (a), avec une nette prédominance statistique des formations du type nom + adjectif, et nom + syntagme prépositionnel.

On rencontre ces locutions non seulement sur leur terreau natal, c'est-à-dire dans les domaines de spécialités, mais encore dans les médias qui les banalisent. Le discours ambiant est ainsi imprégné d'un vernis de technicité, qui vire parfois à l'enflure verbale.

Dans leur domaine de spécialité, leur statut de locution nominale ne fait pas de doute car elles répondent à un besoin de dénomination et renvoient à un référent spécifique. Le critère formel habituel qui distingue ces locutions de simples associations discursives est l'absence d'actualisateurs à l'intérieur du groupe. Le second élément est virtualisé et ne peut donc être accompagné d'aucun déterminatif. Si ces derniers apparaissent, 
c'est qu'il ne s'agit pas de locutions, mais d'associations discursives momentanées. Par exemple, une table ronde et une table bien ronde, ce n'est pas du tout la même chose.

L'élément satellisé est donc engagé dans un processus de virtualisation ou de lexicalisation qui ne se déclenche que par la motivation d'un besoin de dénomination: un appel terminologique. Faute de quoi, on reste dans le domaine de la prédication.

table ronde est une locution.

table imaginative est un effet de rhétorique (on peut appeler ainsi la table d'un grand cuisinier). On reste dans le discours sans atteindre le stade de la lexicalisation.

\section{LABILITÉ DU MODE DE FORMATION}

Les locutions nominales de spécialités sont un domaine mouvant en raison de leur mode de formation: elles passent progressivement du stade de l'association discursive «fortuite» à celui de la locution lexicalisée. Cette idée d'un classement en quelque sorte «génétique» des locutions nous a été suggérée par les réflexions de Benveniste (1974) sur la composition nominale vue comme une «micro-syntaxe»:

Où est la source commune des composés et d'où provient la diversité de leurs formes? Pour répondre à cette question, il faut, à notre avis, envisager les composés non plus comme des espèces morphologiques, mais comme des organisations syntaxiques. La composition nominale est une micro-syntaxe.

Ainsi, il semble que chaque locution nominale pourrait être étudiée comme la transformation d'un énoncé syntaxique libre. Elle se situerait alors le long d'un axe orienté qui irait de l'association discursive à la synthèse morphologique. Voilà pourquoi il est si souvent délicat de repérer une locution pour l'insérer dans un dictionnaire de spécialité. En effet, outre le critère de repérage proprement linguistique évoqué plus haut, il faut maintenant ajouter un critère déterminant: l'usage. Une locution sera dite «en émergence» à un moment donné : le nombre de ses occurrences sera insignifiant du point de vue statistique. Il se peut que, plus tard, son emploi se généralise. Elle sera dite alors «majoritaire». C'est-à-dire que lorsque l'alternance sera linguistiquement possible entre la forme analytique et la locution, les occurrences de la locution seront statistiquement majoritaires. Cette locution peut alors figurer dans un dictionnaire de spécialité.

centre muséal est en émergence, sur le modèle centre expérimental;

centre commercial est majoritaire. Il figure dans le Dictionnaire des termes officiels de la langue française (édition 1994);

centre de gravité est totalement lexicalisé.

Il apparaît que fonder en syntaxe une locution nominale, c'est la placer le long d'un axe, selon le degré de cohésion des signes agencés. Un lien plus ou moins lâche entre les signes agencés situe le syntagme du côté de la syntaxe. Un lien plus ou moins étroit le situe du côté de la morphologie. Le long de cet axe que l'on peut appeler l'axe de la lexicalisation se joue le passage des associations discursives aux synthèses morphologiques.

Ainsi, du côté de la syntaxe, se trouvent des constructions prédicatives actualisées en discours, et du côté de la morphologie, on observe une sorte de marche à la virtualisation menant à la lexicalisation de ces constructions. Entre les deux pôles de ce continuum se placent successivement, et en allant des associations discursives aux synthèses morphologiques:

- les locutions du type: zone de chalandise, gel des salaires;

- puis les locutions du type : table ronde et axe piétonnier. On note une première étape dans la marche à la virtualisation, avec la suppression de la préposition et l'apparition de l'adjectif de relation;

- enfin, les locutions du type: lave-vaisselle. 
Prenons l'exemple de deux locutions que l'on rencontre en alternance libre: gel des salaires et gel salarial. Au-delà de la relation prédicative: «on gèle les salaires», se trouve une première nominalisation: gel des salaires. Une nouvelle étape dans la synthèse morphologique donne: gel salarial. L'adjectif de relation n'exprime nullement un rapport d'inhérence, mais établit, comme son nom l'indique, une simple relation entre deux notions en «condensant» davantage la relation syntaxique. Il représente une étape supplémentaire de virtualisation par rapport à la construction prépositionnelle.

Les cas de non alternance confirment ceci : gel salarial alterne avec gel des salaires. En revanche, axe piétonnier ne peut alterner avec l'association syntagmatique fortuite: axe des piétons. C'est que axe piétonnier est plus engagé que la construction prépositionnelle dans le processus de lexicalisation, qui est aussi un processus de virtualisation.

La lexicalisation des locutions est un processus de virtualisation syntaxique, c'est aussi bien sûr un processus de réduction sémique. La réduction sémique est un procédé commode pour créer de façon économique de nouvelles unités de dénomination. Dans la locution table ronde, l'adjectif est délesté de sa richesse sémique pour pouvoir s'associer à table et désigner ainsi un concept spécifique. Libéré de l'emprise de son «capteur», l'adjectif retrouve toute sa richesse sémique. La réduction sémique est plus ou moins drastique, mais elle est toujours à l'wuvre. Elle est faible dans axe piétonnier, car piétonnier n'est qu'un adjectif de relation, destiné à entrer en locution. En revanche, elle est forte dans table ronde car ronde est un adjectif très riche en sèmes. Lorsque de tels adjectifs entrent en locution, c'est souvent par métaphorisation, comme si l'adjectif trop riche en sèmes devait soit passer par le filtre de la réduction sémique, soit s'alléger vers la métaphore. Cette métaphorisation n'est indispensable qu'aux adjectifs polysémiques qui s'en vont ainsi, à marche forcée, vers la virtualisation.

Il apparaît donc que l'élément déterminatif se virtualise en entrant dans une étroite dépendance, tant syntaxique que sémantique, vis-à-vis de l'élément déterminé. C'est ainsi que l'ensemble acquiert une signification nouvelle par rapport à chaque élément pris séparément.

\section{BANALISATION ET LABILITÉ D'EMPLOI}

Les locutions ne se cantonnent pas dans leurs spécialités. Elles ont tendance à essaimer dans la langue courante où l'on constate un phénomène de banalisation d'une partie des terminologies de spécialités. Outre le fait d'en modifier le sens, la langue courante et celle des médias peuvent les réutiliser à leur façon: elles en reprennent les modes de formation pour les appliquer à des pseudo-locutions qui donnent un vernis de scientificité et d'objectivité au discours. On aboutit de la sorte à des syntagmes parfois proches de l'enflure verbale du style la crise organisationnelle. Si ces tournures sont insérées dans des tours syntaxiques particuliers, nous entrons dans le domaine de la langue de bois. Il est donc nécessaire de pouvoir établir la différence entre une locution de spécialité et ce qui veut lui ressembler et qui appartient au domaine de la rhétorique à la mode. La fiabilité du dictionnaire est en jeu.

crise d'ébullition est une locution du domaine de la thermodynamique.

crise organisationnelle ne répond à aucun appel terminologique. C'est une forme creuse qui entre en alternance avec son équivalent analytique : crise de l'organisation. On reste dans les deux cas dans le domaine de l'agencement syntaxique et de la prédication, en aucun cas dans celui de la synthèse morphologiquc, malgré les apparences formelles. 
Conséquences: au vu des caractéristiques linguistiques et extra-linguistiques des locutions de spécialités, en particulier de leur labilité de formation et d'emploi, il apparaît qu'un dictionnaire de spécialité doit comporter des procédures spécifiques pour l'identification de ces locutions. La diversification des méthodes dictionnairiques ici envisagée découle de la remise en question théorique et de la redéfinition du statut des locutions de spécialités.

Note

* Cet article est issu d'une communication présentée par l'auteur aux IVes Journées scientifiques du réseau «Lexicologie, terminologie, traduction» de l'AUPELF-UREF (Lyon, France, 28, 29, 30 septembre 1995). 